\title{
Coding of PPM Based Modulation Techniques to Improve the Performance of Infrared WLAN's
}

\author{
Rui Alves \\ Instituto de Telecomunicações \\ Campus Universitário Santiago, \\ P-3810 Aveiro, Portugal \\ (rpedro@av.it.pt)
}

\author{
Atílio Gameiro \\ Dep. Electrónica e Telecomnicações \\ Universidade de Aveiro \\ P-3810 Aveiro, Portugal \\ (atilio@av.it.pt)
}

\begin{abstract}
In this communication we consider the use of PPM based modulation methods, such as the hybrid modulation method called Amplitude and Pulse-Position Modulation (APPM) and Overlapping Pulse-Position Modulation (OPPM), to improve the performance of Infrared WLAN's. The bandwidth efficiency expressions of these modulation schemes are presented and compared against the one of Pulse-Position Modulation (PPM), which allows to conclude that both APPM and OPPM can be used to improve the performance of PPM without significant bandwidth expansion, using Trellis-Coded Modulation (TCM) codes. We also derive the best codes for some APPM and OPPM schemes, and the results show that non-negligible coding gains without bandwidth expansion can be obtained with trellis codes of moderate complexity.
\end{abstract}

\section{Introduction}

There has been in recent years, considerable work on InfraRed (IR) links for WLAN's [1]. The performance and capacity coverage of IR WLAN's is highly dependent on the modulation and coding techniques used. In this communication we analyze and compare the following systems: uncoded PPM and Trellis-Coded Modulation (TCM) using PPM based modulation techniques. The PPM based modulation techniques we consider are:

- APPM (Amplitude and Pulse-Position Modulation) [2], which is an hybrid modulation where information is conveyed both in amplitude and position of one pulse.

- OPPM (Overlapped Pulse Position Modulation) [3] where the symbols contain a pulse transmitted in non-orthogonal positions, i.e. allowing some overlapping between pulses of different symbols.
The communication is outlined as follows. In the next section we present the system model and the modulation schemes we will be dealing with. In section 3 we present the expressions for the power spectral density, in order to compare the APPM and OPPM bandwidth efficiencies against PPM. In section 4 we consider trellis coding with $\mathrm{A} \times \mathrm{M}-\mathrm{APPM}$ and M-OPPM, present the optimum codes for some values of the code memory, and evaluate the performance improvement relatively to uncoded 2-PPM. Finally in section 5 we present the main conclusions of this work.

\section{System Model}

The block diagram of the system we are concerned with is shown in Figure 1. The input data sequence $\left\{a_{k}\right\}$ is encoded, modulated and converted to the optical power signal $s(t)$. The channel adds background radiation, which is modeled by an additive noise source $n_{b}(t)$. At the receiver the photodiode converts the incoming optical power $p_{i}(t)=p_{r}(t)+n_{b}(t)$ into an electrical current $z(t)$, which is given by the product of its responsivity $\Re$ multiplied by the optical power integrated over the detector surface. After that, the signal is amplified, demodulated and decoded, giving the output data sequence $\left\{b_{k}\right\}$, that is a replica of the sequence $\left\{a_{k}\right\}$ except for some positions where the decoder was not able to correct channel errors. The coding / modulation operations at the transmitter and decoding / demodulation at the receiver were grouped into single blocks (dashed) to accommodate the use of TCM where the operations are not separated.

In this communication we consider that the received signal does not suffer a significant distortion and the elementary optical pulses received are rectangular. Moreover, the optical intensity modulation with direct detection (IM/DD) system is normally modeled by a signal dependent, Poisson rate, photon-counting model, 


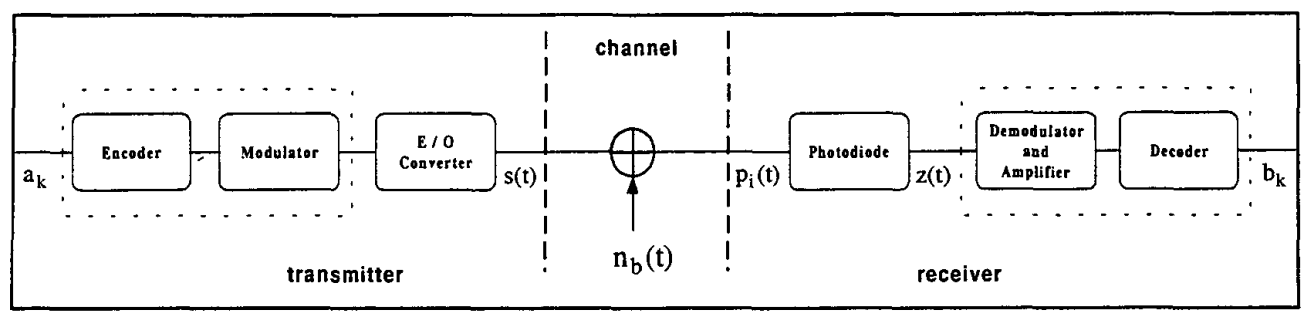

Figure 1. Block diagram of the transmission system.

but for $\mathrm{IR}$ communications due to the intense ambient light in indoor environments the model can be simplified to lead to an AWGN channel. The information bearing electrical current can then be described by:

$$
z(t)=\Re \cdot p_{r}(t)+n(t),
$$

where $p_{r}(t)$ is the received optical power in the absence of noise, $n(t)$ is the additive noise current which is the sum of the photodetector background radiation and thermal noise introduced by the receiver. The noise can be accurately modeled as white Gaussian with power spectral density $n_{o} / 2$.

Assuming that the elementary received pulses are rectangular, the optical received power is given by:

$$
p_{r}(t)=\sum p_{k}\left(t-k T_{s}\right)
$$

where $T_{s}$ is the symbol duration, and $p_{k}(t)$ is the waveform corresponding to the symbol $k$.

\subsection{Modulation Formats}

The modulation formats we will consider are PPM, APPM and OPPM, which will be described in detail next.

\section{M-PPM (Pulse Position Modulation)}

In $M$-PPM, each word of $k$ bits is mapped into one of $M=2^{k}$ symbols and transmitted to the channel. The symbol interval is divided into $M$ chips, and each symbol uses a different chip to transmit a pulse. Thus, the $M$ possible waveforms are: $p_{k}(t)=P_{p} p_{T_{c}}\left(t-i T_{c}\right)$ with $\mathrm{i} \in\{0,1, \ldots, \mathrm{M}-1\}$, and $p_{T_{c}}(t)$ being a rectangular pulse with unit amplitude and chip duration $T_{c}=T_{s} / M$.

The average power is related with the peak power by $P_{a v}=P_{p} / M$.

Detection of M-PPM symbols requires the estimation of the chip where the pulse was most probably transmitted. In the absence of multipath distortion, an optimum maximum-likelihood receiver employs a continuous time filter matched to one chip, whose output is sampled at the chip rate. Each block of $M$ samples is passed to a block decoder, which makes a symbol decision, yielding $k=\log _{2} M$ information bits.

\section{AXM-APPM (Amplitude Pulse Position Modulation)}

In an AxM-APPM symbol there are $M$ chips of $T_{c}$ duration as in M-PPM. The difference from the PPM symbols is that the impulse may take one of the $A$ possible amplitude values, thus, the $A \times M$ different symbol waveforms are: $p_{k}(t)=j P_{p} p_{T_{c}}\left(t-i T_{c}\right)$, with $j \in\{1,2, \cdots, A\}$ and $i \in\{0,1, \ldots, M-1\}$.

The average power is related with the peak power by $P_{c v}=P_{p} \times(1+A) / 2 M$.

It is clear that going from M-PPM to, for example $2 \times M-A P P M$ provides an expansion of the signal set size by a factor of 2 , without in a first order analysis a bandwidth expansion since the duration of the elementary chips remain the same.

\section{$\left(\begin{array}{l}n \\ w\end{array}\right)$ M-OPPM (Overlapping Pulse Position Modul.)}

Overlapping PPM [3] allows multiple positions per pulsewidth, as well as fractional modulation indices (number of pulsewidths per frame) requiring more refined timing than that needed for conventional disjoint PPM. The symbol interval $T_{s}$ is divided into $n$ subintervals (referred as chips) of equal duration $T_{c}$. The information is conveyed by the position of a pulse of duration $T_{p}=w T_{c}$, starting in one of the $M$ instants $t_{k}=(k-1) T_{c}, k=1,2, \ldots, M$, where $t_{1}=0$ is the start of a symbol interval. It is clear that $M$ is related to $n$ and $w$ by: $M=n-w+1$. Thus, the $M$ waveforms allowed for M-OPPM symbols are: $p_{k}(t)=P_{p} p_{T_{p}}\left(t-i T_{c}\right)$ with $i \in\{0,1, \ldots, M-1\}$, and $T_{c}=T_{s} / n$ being the chip duration.

The average power is related with the peak power by $P_{a v}=P_{p} \times w / n$.

Notice that $Q=n / w$ is the alphabet size of the PPM signal set with no overlap and that by allowing overlap between pulses we have increased the number of signals from $Q$ to $M$. The pulsewidth of the OPPM signal is kept the same as that for $Q$-ary PPM. On the other hand, the extended signal set is no longer orthogonal, which implies worse error-probability performance for uncoded transmission systems. 
To obtain efficient OPPM schemes, $(n w)$ must be chosen, so that $M$ is a power of 2 . For example: $(52),(6$ 3) and (7 4) for $M=4$ or (92), (103) for $M=8$.

\section{Bandwidth Efficiency}

The bandwidth of a modulation scheme based on pulse position can be roughly approximated by the inverse of the shortest pulse width. Thus, the bandwidth required by OOK-NRZ is roughly the bit rate $R_{b}$, and the bandwidth required by M-PPM, OPPM and AXM-APPM to achieve a bit rate $R_{b}$ is the inverse of the duration of the shortest pulse, or $M R_{b} / \log _{2} M,(n / w) R_{b} / \log _{2} M \quad$ and $M R_{b} / \log _{2} M$, respectively. More accurately, it is common to specify the bandwidth $B_{x}$ that includes $\mathrm{x} \%$ of the signal power.

Under the assumption that the codewords are chosen independently and with equal probability, the continuous part of the power spectral density functions $S(f)$, are given by:

\section{M-PPM [4]}

$$
S_{P P M}(f)=\frac{P_{p}^{2}}{T_{s}} \operatorname{sinc}^{2}\left(f \frac{T_{s}}{M}\right)\left[1-\left|\frac{1}{M} \frac{\sin \left(\pi f T_{s}\right)}{\sin \left(\pi f T_{s} / M\right)}\right|^{2}\right]
$$

\section{A $\times$ M-APPM [5]}

$$
S_{A P P M}(f)=\frac{P_{p}^{2}}{(A M) T_{s}} \operatorname{sinc}^{2}\left(f \frac{T_{s}}{M}\right)\left[M \sum_{i=1}^{A} i^{2}-\frac{\left|\sum_{i=1}^{A} i\right|^{2}}{(A M)}\left|\frac{\sin \left(\pi g T_{s}\right)}{\sin \left(\pi f T_{s} / M\right)}\right|^{2}\right]
$$

\section{M-OPPM [4]}

$$
S_{O P P M}(f)=\frac{A^{2}}{T_{s}} \operatorname{sinc}^{2}\left(f \frac{w}{n} T_{s}\right)\left\{1-\left|\frac{1}{M} \frac{\sin \left(M \pi f^{T} / n\right)}{\sin \left(\pi f^{T_{s}} / n\right)}\right|^{2}\right\}
$$

where for all equations, $T_{s}$ is the symbol duration.

To perform a more accurate comparison, in what concerns the bandwidth efficiency, we computed for each modulation method the parameter $B_{90}$. This gives the bandwidth value below which $90 \%$ of the continuous power spectral density is contained. The results are presented in Table 1, as well the $B_{N U L L}$ parameter, which is the lowest bandwidth value where the power spectrum is null and, in fact, it equals the inverse of the pulse duration used by each modulation scheme. Both parameters are normalized, i.e. multiplied by the symbol duration $T_{s}$. Table 1 also indicates the number of

\begin{tabular}{|c|c|c|c|}
\hline Modulation & $N^{0}$ Symbols & $\mathbf{B}_{90 .} \mathbf{T}_{\mathrm{s}}$ & B $_{\text {NULL. }} T_{\mathrm{s}}$ \\
\hline M-PPM: & $\mathbf{M}$ & & $\mathbf{M}$ \\
\hline 2-PPM & 2 & 3.05 & 2 \\
\hline 4-PPM & 4 & 5.54 & 4 \\
\hline 8-PPM & 8 & 10.21 & 8 \\
\hline 16-PPM & 16 & 18.97 & 16 \\
\hline (n w) OPPM: & $n-w+1$ & & $\mathbf{n} / \mathbf{w}$ \\
\hline$(52)$ & 4 & 3.81 & 2.5 \\
\hline (6 3) & 4 & 3.44 & 2 \\
\hline$(74)$ & 4 & 4.34 & 1.75 \\
\hline$(92)$ & 8 & 6.15 & 4.5 \\
\hline (10 3) & 8 & 5.01 & 3.33 \\
\hline (114) & 8 & 4.38 & 2.75 \\
\hline (125) & 8 & 4.15 & 2.4 \\
\hline$(136)$ & 8 & 4.99 & 2.16 \\
\hline AXM-APPM: & $\mathbf{A} \times \mathbf{M}$ & & $\mathbf{M}$ \\
\hline 2×2-APPM & 4 & 2.96 & 2 \\
\hline 4×2-APPM & 8 & 2.91 & 2 \\
\hline 2×4-APPM & 8 & 5.36 & 4 \\
\hline 4×4-APPM & 16 & 5.30 & 4 \\
\hline
\end{tabular}
symbols, i.e. the alphabet cardinality for the different modulation schemes.
Table 1. $B_{90}$ and $B_{N U L L}$ for different modulation schemes.

We can observe from the comparison between $B_{90}$ and $B_{N U L L}$, not only their disparity but mainly that, while the latter one gives a very optimistic estimation for the bandwidth requirements of a certain modulation scheme, $B_{90}$ gives a more realistic estimation of the required bandwidth. Thus, in the next sections we will use $B_{90}$, to assess the bandwidth of TCM coded signals using APPM and OPPM modulations.

\section{TCM codes for APPM and OPPM}

It is well known that the classical separation of coding and modulation requires some bandwidth expansion. When the channel is bandwidth constrained, this may be unacceptable. To illustrate ideas let us consider the use of PPM with a $1 / 2$ convolutional code. In the absence of coding, the chip duration of the $2^{k}$-PPM pulse is related to the bit duration through:

$$
T_{c}=\frac{k}{2^{k}} \cdot T_{b},
$$

while with the use of the $1 / 2$ convolutional code this goes to:

$$
T_{c}=\frac{k}{2 \cdot 2^{k}} \cdot T_{b}
$$

If one is bandwidth limited, and using the first order approximation that the PPM bandwidth is proportional to the inverse of the chip rate, then one has to reduce the PPM order to accommodate coding. Performing the calculations, one finds for example that, to maintain the bandwidth we should go from 16-PPM to 4-PPM or from 64-PPM to 16-PPM. Asymptotically this represents a 


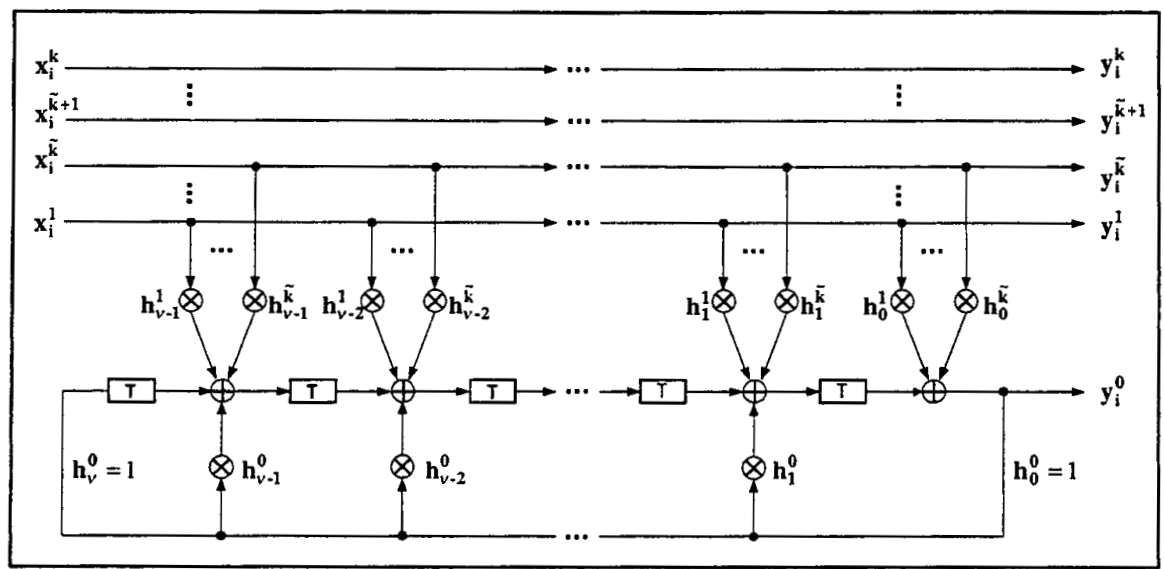

Figure 2. General structure of systematic feedback convolutional encoder with $\mathrm{k} /(\mathrm{k}+1)$ efficiency and memory $v$ with $\tilde{k}$ checked bits.

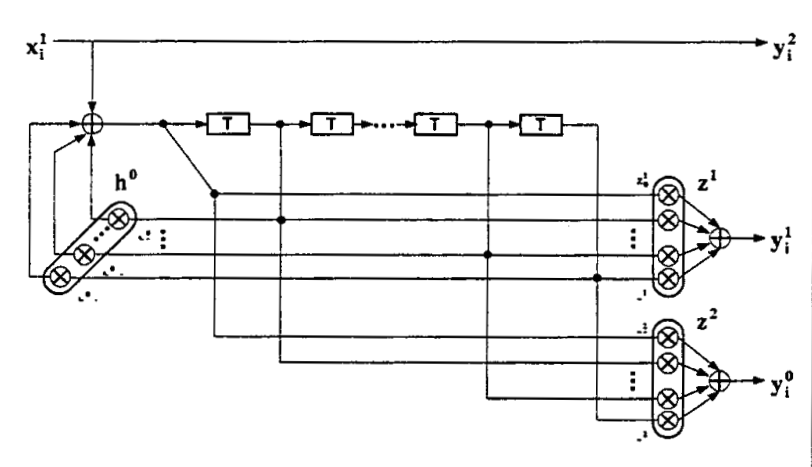

Figure 3. Structure of systematic $1 / 3$ feedback convolutional encoder with memory $v$.

power penalty through the reduction of the set dimensionality of approximately 3 optical $\mathrm{dB}$, which implies that the convolutional code must give at least $3 \mathrm{~dB}$ coding gain with hard decoding.

This means that if bandwidth is a constraint, one should combine the modulation and coding schemes according to TCM. So, it was already pointed out at [2] that $\mathrm{A} \times \mathrm{M}-\mathrm{APPM}$ represents a method to augment the alphabet size of M-PPM without bandwidth expansion. In this communication we intend to enlarge this conclusion to OPPM.

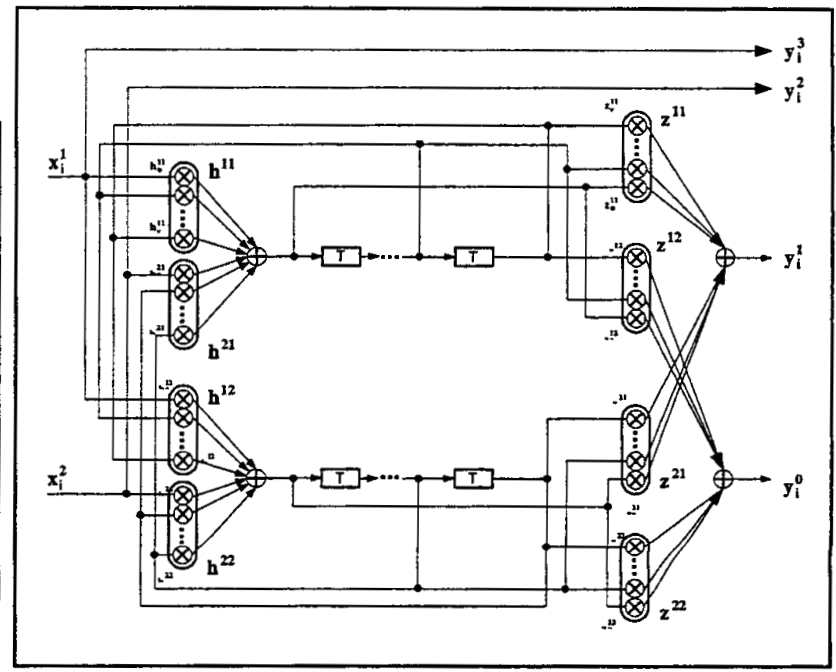

Figure 4. Structure of systematic $2 / 4$ feedback convolutional encoder with memory $v$.

\subsection{Coding Structures}

In order to find out efficient TCM codes for APPM and OPPM schemes, we considered convolutional encoders with $1 / 2,2 / 3,1 / 3$ and $2 / 4$ rates. The $1 / 2$ encoders are used by schemes with 4 symbols, $1 / 3$ and $2 / 3$ encoders by schemes with 8 symbols and $2 / 4$ by schemes with 16 symbols.

Figures 2,3 and 4 illustrate the structures for the considered encoders. The $1 / 2$ and $2 / 3$ convolutional encoders are particular cases of the general structure for $\mathrm{k} /(\mathrm{k}+1)$ systematic convolutional encoder with feedback. 
This is represented in Figure 2 as proposed in [6] for memory $v$ and $\tilde{k}$ checked bits (bits used to calculate the coded output bit $y_{i}^{0}$ ) The structure for the encoder with rate $1 / 2$ and memory $v$ is obtained, making $k=1$ e $\tilde{k}=1$, thus the encoder is defined by its two polynomials $h^{0}$ with $v+1$ binary coefficients, and $h^{1}$ with $v$ coefficients. For the $2 / 3$ convolutional encoder we consider $k=2$ and $\tilde{k}=2$, and the encoder is defined by three polynomials: $h^{0}, h^{1}$ and $h^{2}$. When $h^{1}=0$ or $h^{2}=0$, but $h^{1} \neq h^{2}$ the structure degenerates to $k=2$ and $\tilde{k}=1$. In the exhaustive search we performed, no restriction was made on $h^{2}$ and then this situation was accommodated. In fact the results that will be presented later, show that for $2 \times 4$-APPM the optimum encoders lead to $h^{2}=0$.

In Figure 3 we present the structure of a $1 / 3$ systematic feedback convolutional encoder with memory order $v$, suggested by Benedetto in [7]. This encoder is defined by the three polynomials: $h^{0}$ with $v$ coefficients, and $z^{1}$, $z^{2}$ both with $v+1$ coefficients.

Figure 4 shows the structure of a $2 / 4$ systematic feedback convolutional encoder with memory order $v$, also suggested in [7]. This structure is based on two independent rows of memory elements with orders $p_{1}$ and $p_{2}$, whose sum equals the encoder memory $v$. Thus, this convolutional encoder is defined by 8 polynomials: 4 $\left(h^{11}, h^{12}, z^{11}\right.$ and $\left.z^{12}\right)$ related to the upper memory row, and $4\left(h^{21}, h^{22}, z^{21}\right.$ and $\left.z^{22}\right)$ related to the lower memory row. The former ones have $p_{1}+1$ coefficients, while the latter have $p_{2}+1$ coefficients.

\subsection{Results}

\section{Asymptotic Coding Gains}

In order to assess the merits of coding each modulation scheme, we must search the TCM codes that maximize the gains. For the desired infrared link applications working on high SNRs and having low error-probabilities, the asymptotic coding gain is a good approximation to the real coding gain achieved by TCM codes. The asymptotic coding gain of a TCM coded modulation scheme, relatively to uncoded $2-P P M$ transmission is given by:

$$
G_{\text {cod }}=10 \log \left[\frac{\left(d_{\text {free }}^{2} / \bar{P}_{c o d}\right)}{\left(d_{u n c}^{2} / \bar{P}_{u n c}\right)}\right]
$$

where $d_{\text {free }}^{2}$ is the squared minimum Euclidean distance between all coded sequences, $\bar{P}_{\text {cod }}$ is the mean power of constellation signals that constitute the modulation alphabet, $d_{u n c}^{2}$ is the squared minimum Euclidean distance between 2-PPM symbols and $\bar{P}_{\text {unc }}$ is the mean power of 2-PPM alphabet. If we normalize the amplitude of 2-PPM pulses to 1 , we obtain $\bar{P}_{\text {unc }}=1 / 2$ and $d_{\text {unc }}^{2}=2$. In Table 3 we present the respective asymptotic coding gain expressions used for each APPM and OPPM modulation schemes considered.

Hence, search for the best codes is equivalent to find out the codes with maximum asymptotic coding gains. From (8) we can conclude that the asymptotic coding gain maximization for a given modulation scheme, is equivalent to search the convolutional encoder with maximal $d_{\text {free }}^{2}$. This was the criterion used to find the best TCM codes, which were obtained by performing an exhaustive computational search.

After exhaustive inspection, we noted that all considered TCM codes using any APPM or OPPM modulation schemes, belong to the symmetry class of quasi-regular codes (QRC or ZWC) by verifying the Zehavi-Wolf condition [8]. So, to proceed with $d_{\text {free }}^{2}$ evaluation, the GUZWA algorithm [8] was used.

Another important aspect is concerned with the modulation partition. It was necessary to do a previous selection of the best signal constellation partition for each TCM code (different modulation scheme or different convolutional encoder rate). This was not a trivial task and the criterion used was to select the partition that maximizes the summation of the distance profile used by GUZWA algorithm. For partitions with equal summation of the distance profile, exhaustive searches were only made for codes of small memory orders (typically up to 4), while for high orders (5 and 6), the search was restricted to the partition that had already given the best results. This methodology does not ensure that the optimum TCM codes were found, but we guarantee good ones.

In Table 2, we present the results of this search: the best free distance attained and one of the best codes found, for each modulation and for memory values between 2 and 6 . The polynomials that define the different encoders are indicated in Table 2 using octal notation followed by Pietrobon in [6]. As an example: $h^{0}=\left(h_{4}^{0}, h_{3}^{0}, h_{2}^{0}, h_{1}^{0}, h_{0}^{0}\right)_{2}=(0,1,1,0,1)_{2}$ that is denoted in

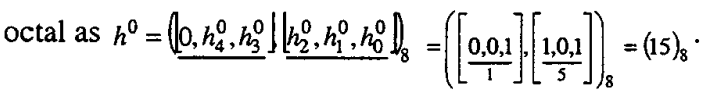

In Table 3, we present the asymptotic power gains, achieved by using the best codes for each considered $\mathrm{A} \times \mathrm{M}-\mathrm{APPM}$ and $(n w)-\mathrm{OPPM}$ schemes, relatively to uncoded 2-PPM. 


\begin{tabular}{|c|c|c|c|c|c|c|c|}
\hline \multirow{2}{*}{ Modulation } & \multirow{2}{*}{$\begin{array}{c}\text { Encoder } \\
\text { Rate }\end{array}$} & \multirow{2}{*}{ Code } & \multicolumn{5}{|c|}{ Encoder Memory Order } \\
\hline & & & 2 & 3 & 4 & 5 & 6 \\
\hline \multirow{2}{*}{$2 \times 2-\mathrm{APPM}$} & \multirow{2}{*}{$1 / 2$} & $\overline{d_{\text {free }}^{2}}$ & 12 & 14 & 16 & 17 & 19 \\
\hline & & $\left(h^{0} ; h^{1}\right)$ & $(7 ; 2)$ & $(15 ; 2)$ & $(31 ; 02)$ & $(45 ; 12)$ & $(187 ; 32)$ \\
\hline \multirow{2}{*}{$4 \times 2-\mathrm{APPM}$} & \multirow{2}{*}{$1 / 3$} & $d_{\text {free }}^{2}$ & 31 & 36 & 42 & 46 & 53 \\
\hline & & $\left(h^{0} ; z^{1} ; z^{2}\right)$ & $(3 ; 2 ; 5)$ & $(6 ; 02 ; 17)$ & $(16 ; 12 ; 27)$ & $(36 ; 16 ; 63)$ & $(71 ; 056 ; 135)$ \\
\hline \multirow{2}{*}{$2 \times 4-A P P M$} & \multirow{2}{*}{$2 / 3$} & $d_{\text {free }}^{2}$ & 12 & 15 & 17 & 19 & 21 \\
\hline & & $\left(h^{0} ; h^{1} ; h^{2}\right)$ & $(7 ; 1 ; 0)$ & $(13 ; 1 ; 0)$ & $(23 ; 11 ; 00)$ & $(73 ; 11 ; 00)$ & $(133 ; 11 ; 00)$ \\
\hline \multirow{5}{*}{ 4×4-APPM } & \multirow{5}{*}{$2 / 4$} & $d_{\text {free }}^{2}$ & 31 & 38 & 42 & 48 & 53 \\
\hline & & $\left(h^{11} ; h^{12}\right)$ & $(2 ; 1)$ & $(4 ; 0)$ & $(4 ; 4)$ & $(10 ; 10)$ & $(10 ; 00)$ \\
\hline & & $\left(h^{21} ; h^{22}\right)$ & $(2 ; 3)$ & $(2 ; 1)$ & $(4 ; 7)$ & $(7 ; 4)$ & $(10 ; 01)$ \\
\hline & & $\left(z^{11} ; z^{12}\right)$ & $(2 ; 0)$ & $(0 ; 0)$ & $(0 ; 6)$ & $(01 ; 03)$ & $(00 ; 10)$ \\
\hline & & $\left(z^{21} ; z^{22}\right)$ & $(2 ; 3)$ & $(1 ; 3)$ & $(3 ; 1)$ & $(3 ; 7)$ & $(01 ; 03)$ \\
\hline \multirow{2}{*}{$\left(\begin{array}{l}5 \\
2\end{array}\right)-\mathrm{OPPM}$} & \multirow{6}{*}{$1 / 2$} & $d_{\text {free }}^{2}$ & 4.00 & 4.80 & 5.60 & 6.40 & 8.00 \\
\hline & & $\left(h^{0} ; h^{1}\right)$ & $(5 ; 2)$ & $(15 ; 2)$ & $(31 ; 02)$ & $(55 ; 02)$ & $(117 ; 42)$ \\
\hline \multirow{2}{*}{$\left(\begin{array}{l}6 \\
3\end{array}\right)-O P P M$} & & $d_{\text {free }}^{2}$ & 3.33 & 4.00 & 4.67 & 5.33 & 6.67 \\
\hline & & $\left(h^{0} ; h^{1}\right)$ & $(5 ; 2)$ & $(15 ; 2)$ & $(31 ; 02)$ & $(55 ; 02)$ & $(117 ; 42)$ \\
\hline \multirow{2}{*}{$\left(\begin{array}{l}7 \\
4\end{array}\right)-O P P M$} & & $d_{\text {free }}^{2}$ & 2.86 & 3.43 & 4.00 & 4.57 & 5.71 \\
\hline & & $\left(h^{0} ; h^{1}\right)$ & $(5 ; 2)$ & $(15 ; 2)$ & $(31 ; 02)$ & $(55 ; 02)$ & $(117 ; 42)$ \\
\hline \multirow{2}{*}{$\left(\begin{array}{l}9 \\
2\end{array}\right)-O P P M$} & \multirow{8}{*}{$1 / 3$} & $d_{\text {free }}^{2}$ & 2.67 & 3.56 & 4.44 & 5.33 & 6.22 \\
\hline & & $\left(h^{0} ; z^{1} ; z^{2}\right)$ & $(0 ; 1 ; 6)$ & $(1 ; 03 ; 15)$ & $(02 ; 05 ; 32)$ & $(05 ; 13 ; 65)$ & $(22 ; 045 ; 133)$ \\
\hline \multirow{2}{*}{$\left(\begin{array}{c}10 \\
3\end{array}\right)-O P P M$} & & $d_{\text {free }}^{2}$ & 3.20 & 4.40 & 5.20 & 6.00 & 6.80 \\
\hline & & $\left(h^{0} ; z^{1} ; z^{2}\right)$ & $(1 ; 1 ; 7)$ & $(3 ; 05 ; 17)$ & $(16 ; 27 ; 35)$ & $(17 ; 35 ; 57)$ & $(76 ; 123 ; 175)$ \\
\hline \multirow{2}{*}{$\left(\begin{array}{c}11 \\
4\end{array}\right)-O P P M$} & & $d_{\text {free }}^{2}$ & 3.64 & 4.36 & 5.82 & 6.18 & 7.27 \\
\hline & & $\left(h^{0} ; z^{1} ; z^{2}\right)$ & $(2 ; 2 ; 5)$ & $(2 ; 02 ; 15)$ & $(13 ; 12 ; 27)$ & $(30 ; 02 ; 55)$ & $(47 ; 022 ; 117)$ \\
\hline \multirow[t]{2}{*}{$\left(\begin{array}{c}12 \\
5\end{array}\right)-O P P M$} & & $d_{\text {free }}^{2}$ & 3.33 & 4.00 & 5.33 & 5.67 & 6.67 \\
\hline & & $\left(h^{0} ; z^{1} ; z^{2}\right)$ & $(2 ; 2 ; 5)$ & $(2 ; 02 ; 15)$ & $(13 ; 12 ; 27)$ & $(30 ; 02 ; 55)$ & $(47 ; 022 ; 117)$ \\
\hline \multirow{2}{*}{$\left(\begin{array}{l}9 \\
2\end{array}\right)-O P P M$} & \multirow{8}{*}{$2 / 3$} & $d_{\text {free }}^{2}$ & 4.44 & 6.22 & 6.22 & 8.00 & 8.89 \\
\hline & & $\left(h^{0} ; h^{1} ; h^{2}\right)$ & $(7 ; 1 ; 1)$ & $(15 ; 3 ; 3)$ & $(31 ; 02 ; 02)$ & $(67 ; 05 ; 05)$ & $(153 ; 31 ; 31)$ \\
\hline \multirow[t]{2}{*}{$\left(\begin{array}{c}10 \\
-O P P M\end{array}\right.$} & & $d_{\text {free }}^{2}$ & 5.60 & 6.40 & 7.20 & 8.80 & 9.60 \\
\hline & & $\left(h^{0} ; h^{1} ; h^{2}\right)$ & $(5 ; 2 ; 2)$ & $(13 ; 1 ; 1)$ & $(31 ; 02 ; 02)$ & $(67 ; 35 ; 35)$ & $(133 ; 11 ; 11)$ \\
\hline \multirow{2}{*}{$\left(\begin{array}{c}11 \\
4\end{array}\right)-O P P M$} & & $d_{\text {free }}^{2}$ & 5.82 & 6.55 & 7.27 & 8.00 & 8.73 \\
\hline & & $\left(h^{0} ; h^{1} ; h^{2}\right)$ & $(5 ; 2 ; 2)$ & $(15 ; 2 ; 2)$ & $(31 ; 02 ; 02)$ & $(45 ; 02 ; 02)$ & $(131 ; 02 ; 02)$ \\
\hline \multirow{2}{*}{$\left(\begin{array}{c}12 \\
5\end{array}\right)-O P P M$} & & $d_{\text {free }}^{2}$ & 5.33 & 6.00 & 6.67 & 7.33 & 8.00 \\
\hline & & $\left(h^{0} ; h^{1} ; h^{2}\right)$ & $(5 ; 2 ; 2)$ & $(15 ; 2 ; 2)$ & $(31 ; 02 ; 02)$ & $(45 ; 02 ; 02)$ & $(131 ; 02 ; 02)$ \\
\hline
\end{tabular}

Table 2. Optimum TCM codes and obtained free distances for APPM and OPPM modulations. 


\begin{tabular}{|c|c|c|c|c|c|c|c|c|}
\hline \multirow{2}{*}{ MODULATION } & \multirow{2}{*}{$\bar{P}_{c o d}$} & \multirow{2}{*}{$G_{\text {cod }}\left(\mathrm{dB}_{\text {electric }}\right)$} & \multirow{2}{*}{$\begin{array}{l}\text { Encoder } \\
\text { Rate }\end{array}$} & \multicolumn{5}{|c|}{$\begin{array}{c}\text { Asymptotic power gains ( } \mathrm{dB}_{\text {electric }} \text { ) } \\
\text { Encoder memory order }\end{array}$} \\
\hline & & & & 2 & $\frac{1}{3}$ & 4 & 5 & 6 \\
\hline $2 \times 2$-APPM & $5 / 2$ & $10 \log \left(1 / 5 d_{\text {free }}^{2}\right)$ & $1 / 2$ & 3.80 & 4.47 & 5.05 & 5.32 & 5.80 \\
\hline $4 \times 2-A P P M$ & $15 / 2$ & $10 \log \left(1 / 15 d_{\text {free }}^{2}\right)$ & $1 / 3$ & 3.15 & 3.80 & 4.47 & 4.87 & 5.48 \\
\hline $2 \times 4-\mathrm{APPM}$ & $5 / 4$ & $10 \log \left(2 / 5 d_{\text {free }}^{2}\right)$ & $2 / 3$ & 6.81 & 7.78 & 8.32 & 8.81 & 9.24 \\
\hline 4×4-APPM & $15 / 4$ & $10 \log \left(2 / 15 d_{\text {free }}^{2}\right)$ & $2 / 4$ & 6.16 & 7.05 & 7.48 & 8.06 & 8.49 \\
\hline$\left(\begin{array}{l}5 \\
2\end{array}\right)$-OPPM & $w / n=2 / 5$ & \multirow{11}{*}{$10 \log \left(\frac{n}{2 w} d_{\text {free }}^{2}\right)$} & \multirow{3}{*}{$1 / 2$} & 6.99 & 7.78 & 8.45 & 9.03 & 10.00 \\
\hline$\left(\begin{array}{l}6 \\
3\end{array}\right)$-OPPM & $w / n=3 / 6$ & & & 5.23 & 6.02 & 6.69 & 7.27 & 8.24 \\
\hline$\left(\begin{array}{l}7 \\
4\end{array}\right)^{-\mathrm{OPPM}}$ & $w / n=4 / 7$ & & & 3.98 & 4.77 & 5.44 & 6.02 & 6.99 \\
\hline$\left(\begin{array}{l}9 \\
2\end{array}\right)^{-O P P M}$ & $w / n=2 / 9$ & & \multirow{4}{*}{$1 / 3$} & 7.78 & 9.03 & 10.00 & 10.79 & 11.46 \\
\hline$\left(\begin{array}{c}10 \\
3\end{array}\right)-\mathrm{OPPM}$ & $w / n=3 / 10$ & & & 7.27 & 8.65 & 9.38 & 10.00 & 10.54 \\
\hline$\left(\begin{array}{c}11 \\
4\end{array}\right)-O P P M$ & $w / n=4 / 11$ & & & 6.99 & 7.78 & 9.03 & 9.29 & 10.00 \\
\hline$\left(\begin{array}{c}12 \\
5\end{array}\right)$-OPPM & $w / n=5 / 12$ & & & 6.02 & 6.81 & 8.06 & 8.33 & 9.03 \\
\hline$\left(\begin{array}{l}9 \\
2\end{array}\right)^{-\mathrm{OPPM}}$ & $w / n=2 / 9$ & & \multirow{4}{*}{$2 / 3$} & 10.00 & 11.46 & 11.46 & 12.55 & 13.01 \\
\hline$\left(\begin{array}{c}10 \\
3\end{array}\right)$-OPPM & $w / n=3 / 10$ & & & 9.70 & 10.28 & 10.79 & 11.66 & 12.04 \\
\hline$\left(\begin{array}{c}11 \\
4\end{array}\right)^{-O P P M}$ & $w / n=4 / 11$ & & & 9.03 & 9.54 & 10.00 & 10.41 & 10.79 \\
\hline$\left(\begin{array}{c}12 \\
5\end{array}\right)-\mathrm{OPPM}$ & $w / n=5 / 12$ & & & 8.06 & 8.57 & 9.03 & 9.44 & 9.82 \\
\hline
\end{tabular}

Table 3. Asymptotic coding gains for the best TCM codes found with APPM and OPPM modulations relatively to 2-PPM.

Since the photodetector has a squaring effect, it is clear, that the power gains in terms of the received optical power is half of the values reported in Table 3 . Nonetheless one can conclude that quite appreciable gains can be obtained with convolutional encoders of moderate to low memory.

Inspection of Table 3 shows that there is no advantage from increasing the number of amplitude levels in AxMAPPM beyond 2. Concerning OPPM we observe that the best coding gains, for a given $(n w)$ scheme, are achieved with a $\mathrm{k} /(\mathrm{k}+1)$ code.

In order to compare all studied modulations, we plot in Figure 5, for all considered schemes their coding gains against uncoded 2-PPM versus their bandwidth requirements. The points plotted refer to the "bandwidth efficiency/coding gains" figures of the following modulation formats:

- uncoded 2-PPM, which is used as the reference.
- uncoded 4-PPM, which has a normalized bandwidth of 0.91 and a coding gain of $6 \mathrm{~dB}$.

- coded modulation schemes for APPM and OPPM schemes with TCM encoders with memory orders equal to 2,4 and 6 .

It must be noted that for OPPM schemes with 8 symbols, namely (92), (103), (114) and (125), the best performance was obtained with $2 / 3$ codes, and only these ones were plotted in Figure 5. The bandwidth required by each coded modulation scheme when used in a TCM code with rate $k / n$, is given in a fist order approximation by the $\left(B_{90} \cdot T_{b}\right)_{c o d}$ parameter, which is related with the $\left(B_{90} \cdot T_{s}\right)_{u n c}$ values, presented in Table 1 , by the expression:

$$
\left(B_{90} \cdot T_{b}\right)_{c o d}=\left(B_{90} \cdot T_{s}\right)_{u n c} \frac{n}{k \cdot r}
$$




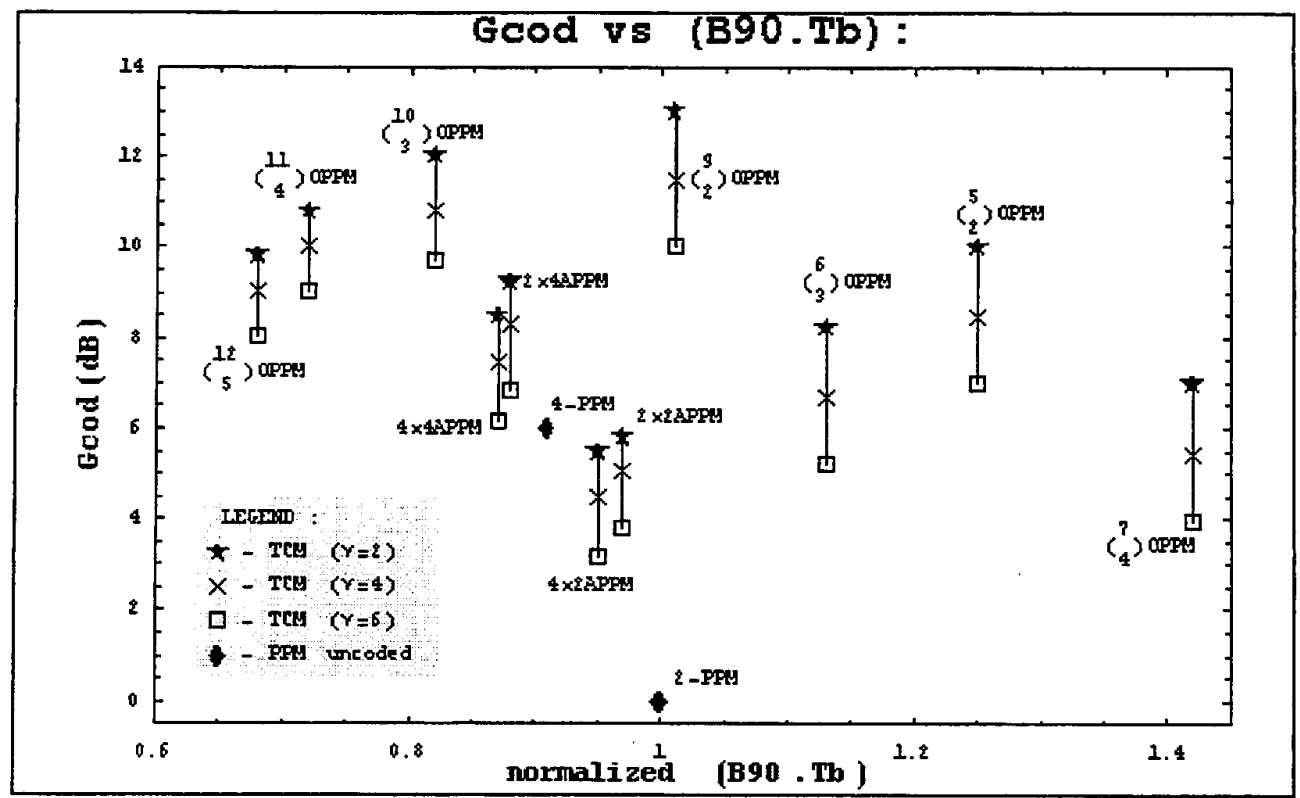

Figure 5. Asymptotic coding gains (Gcod) versus $\left(B_{s o} \cdot T_{b}\right)$ for the various analyzed modulation schemes. The tradeoff: coding gains / bandwidth efficiency, for TCM coding with APPM and OPPM schemes.

where $r$ is the number of bits corresponding to one modulation symbol, i.e. the number of symbols of the modulation scheme is $M=2^{r}$.

We observe that appreciable coding gains are achieved without loss of bandwidth efficiency with AxM-APPM. This improvement may still be enhanced by using some $(n w)$ OPPM schemes. Also concerning OPPM the use of 8 symbols gives significant bandwidth and coding improvements when compared to OPPM of 4 symbols.

\section{Conclusions}

In this communication, the bandwidth efficiencies of APPM and OPPM were derived and compared against the Pulse-Position Modulation (PPM) ones, which allows to conclude that these PPM based modulations can be used to improve the performance of PPM without significant bandwidth expansion, using Trellis-Coded Modulation (TCM) codes. The best codes for some AXM-APPM and ( $n$ w) -OPPM schemes were found and their asymptotic coding gains computed relatively to uncoded 2-PPM. The results show that both APPM and OPPM are quite simple but effective ways of expanding the alphabet size of PPM to apply TCM techniques, and then to improve the performance of wireless infrared communication systems.

\section{References}

[1] J. Kahn, J. Barry, "Wireless Infrared Communications", Proceedings of the IEEE, vol. 85, $\mathrm{n}^{\circ} .2$ 2, Feb. 1997.

[2] R. Alves, A. Gameiro, "Trellis Codes Based on Amplitude and Position Modulation for Infrared WLAN's", VTC '99-Fall Vol.5, pg. 2934-2938, Amsterdam, September 19-22,1999.

[3] C. Georghiades, "Some Implications of TCM for Optical Direct-Detection Channels", IEEE Trans. On Communications, Vol.37, $N^{\circ} 5$, May 1989.

[4] H. Park, J. R. Barry: "Performance of multiple pulse position modulation on multipath channels", IEE Proc.-Optoelectron., Vol. 143, N 6, December 1996.

[5] R. Alves, "Modulation Techniques for IR WLAN's", Universidade de Aveiro, Internal Publication.

[6] S. Pietrobon, R. Deng, A. Lafanechére, G. Ungerboeck, D. Costello: "Trellis-Coded Multidimensional Phase Modulation", IEEE Trans. on Information Theory, Vol. $36, \mathrm{~N}^{\circ} 1$, January 1990.

[7] S. Benedetto, R. Garello and G. Montorsi: "Canonical structure for systematic rate $\mathrm{k} / \mathrm{n}$ convolutional encoders and its application to turbo codes", Electronics Letters, Vol.32, $\mathrm{N}^{\circ} 11$, 23rd May 1996

[8] S. Benedetto, M. Mondim and G. Montorsi: "Performance Evaluation of Trellis-Coded Modulation Schemes", Proceedings of the IEEE, Vol. 82, № 6, June 1994. 